\title{
Artelogie
}

Recherche sur les arts, le patrimoine et la littérature de l'Amérique latine

$11 \mid 2017$

Délocalités, translocalités et activisme dans l'art électronique et biomédiale latino-américain

\section{Introduction $\mathrm{N}^{\circ} 11$ - Délocalités, translocalités et activisme dans l'art électronique et biomédiale latino-américain}

Pat Badani, Iliana Hernandez Garcia, Claudia Kozak et Priscila Arantes

\section{CpenEdition}

Journals

Édition électronique

URL : http://journals.openedition.org/artelogie/1668

DOI : $10.4000 /$ artelogie. 1668

ISSN : 2115-6395

Éditeur

Association ESCAL

Référence électronique

Pat Badani, lliana Hernandez Garcia, Claudia Kozak et Priscila Arantes, «Introduction Nำ1 -

Délocalités, translocalités et activisme dans l'art électronique et biomédiale latino-américain »,

Artelogie [En ligne], 11 | 2017, mis en ligne le 28 décembre 2017, consulté le 24 septembre 2020. URL :

http://journals.openedition.org/artelogie/1668; DOI : https://doi.org/10.4000/artelogie.1668

Ce document a été généré automatiquement le 24 septembre 2020.

Association ESCAL 


\title{
Introduction $\mathrm{N}^{\circ} 11$ - Délocalités, translocalités et activisme dans l'art électronique et biomédiale latino- américain
}

\author{
Pat Badani, Iliana Hernandez Garcia, Claudia Kozak et Priscila Arantes
}

1 Le présent dossier rassemble des articles prenant comme objet les arts électroniques et biomédiales latino-américains en articulation avec un certain nombre de concepts, tels que les délocalités, les translocalités et l'activisme. Ces concepts proposés questionnent à la fois les différentes relations possibles entre les pratiques artistiques et les contextes de production spécifiques et celles entre les pratiques artistiques et les contextes de réception des arts. Ces derniers sont traversés aussi bien par des positionnements "glocals" et "translocals" divers que par des mutations ontologiques que notre époque est en train de produire en relation - non plus avec des localisations régionales - mais avec le vivant, dans un sens plus large. Ce numéro 11 de la revue Artelogie, voudrait également convoquer des propositions artistiques sur l'art et la technologie en lien avec l'activisme impliqué dans la mobilisation et la création de nouvelles écologies sociales issues des actions collectives, relationnelles et collaboratives.

2 Le concept de délocalisation invite à une réflexion sur l'importance de l'extension des discussions, au-delà des contextes historiques bornés. Ce terme ne vise pas pour autant, dans ce cas, d'autres sens étendus, notamment son emploi dans le monde des affaires qui traduit la recherche des marchés où le travail est à moindre coût.

3 Le concept fait référence à des délocalisations posthumaines suivant des logiques trivalentes ou polyvalentes, impliquant des expérimentations artistiques capables de faire face aux nouvelles ontologies non réduites à des binarismes capables d'exclure, eux, de nombreuses ouvertures possibles. Les logiques trivalentes ou polyvalentes sont celles qui considèrent plus de trois possibilités de résolution, en même temps, à un problème d'étude. Par exemple, ontologiquement, dans ses relations avec les 
délocalisations posthumaines, cette logique habilite, en même temps, l'être humain et le non humain, comme l'indiquerait l'existence d'un être machinique. En somme, on est vivant par degrés avec des natures différentes et non à partir d'un jeu d'oppositions. En outre, ces expérimentations artistiques interrogent le problème de la vie - pas nécessairement humain, ou humaniste, ou même terrestre - en parallèle avec des positionnements "glocals" ou "translocals" propres à la théorie de l'art électronique et biomédiale. Pour ne pas tomber dans une nouvelle forme de colonialisme interne à l'Amérique latine, ou externe (avec l'objectif, par exemple, de décoloniser l'Amérique latine), on postule qu'il serait nécessaire de produire, au sein même du continent, de l'art et de la pensée capables de prendre en charge des phénomènes qui concernent les modes généraux d'existence, qui ne seraient pas seulement Latino-américains.

4 Depuis quelques temps, les penseurs de la philosophie contemporaine, comme Peter Sloterdijk, Andy Clark, David Chalmers et Paul Humphreys, entre autres, alimentent le débat au sujet de la « rupture ontologique » («ontological turn») provoquée par la vision des mondes qui coexistent dans une multiplicité relationnelle. Cette approche comprendrait d'une part, une réflexion sur la vie et l'homme coexistant avec l'artificiel et d'autre part, ferait allusion à des systèmes complexes et émergents pour repenser les nouvelles écologies de la vie humaine, et leurs relations avec l'électromécanique. Cet axe d'analyse permettrait d'embrasser de vastes échelles de temps, dans lesquelles on peut remarquer des imprévisibilités et des transformations radicales présentant une puissance créatrice machinique. Ces derniers éléments impliquent forcément l'étude de l'impossible au sein même d'une esthétique qui comprend des fonctions et des modèles non déterministes dans les processus créatifs. Lesdits processus redéfinis peuvent, dès lors, s'employer pour une épistémologie du non humain et pour la création de mondes virtuels immersifs. Ceux-ci sont fabriqués avec des "software ", " hardware » ou " wetware » en évolution, impliquant des considérations sur l'espace/temps délocalisé et sur la matière synthétique.

5 En réalité, ces discussions sont parallèles à des enjeux de translocalité dans l'art contemporain (dans son sens le plus large qui englobe l'art et la technologie) et surviennent dans un contexte de rupture et de questionnements eurocentriques liés aux créations artistiques. À cet égard, nous essayons de comprendre les complexités et les différents regards produits par le dialogue avec les différences, les désencadrements et avec les opérations de créations de mondes agissants, par exemple, comme des productions capables d'activer des espaces sociaux. Dans ce sens, la plupart des discussions en cours dans le domaine des interfaces entre l'art, la science et la technologie sont tout à fait liées à des questions d'activisme. Autrement dit, il s'agit de mettre en lumière la capacité de l'art à fonctionner dans l'espace de la critique, de la manifestation et, surtout, de l'action. Dans cette perspective, le tournant social dans l'art contemporain et dans les arts électroniques («social turn»), ou pour certains, le virage éducatif ( « educational turn»), traite de la compréhension de l'art en tant que processus créatif et pratique créative, et comme un médium capable de renouveler les relations, les connaissances et les écologies humaines et sociales.

6 La combinaison de l'art et de la technologie à l'action sociale, entre les artistes et les non-artistes à partir des pratiques de collaboration, intersubjectives, relationnelles et collectives, implique, dans ce contexte, la compréhension de ces productions, comme des formes actives dans le processus de la connaissance et de la modification de la réalité vivante. La production de l'art et de la technologie est ici conçue moins comme 
un dispositif de la langue à l'intérieur d'une perspective formaliste, que comme un transit et une pratique des nouveaux espaces collaboratifs et participatifs.

7 Afin de répondre à ces points antérieurement délimités, le numéro 11 de la revue Artelogie invite les chercheurs, les artistes et les acteurs des différentes disciplines, liées à la théorie et la production de l'art électronique et biomédiale (littérature électronique, musique électroacoustique, bio-art, art génératif, robotique, ethnographie et sociologie numérique, paléonto-écologie numérique, sciences sociales post-humaines numériques), à envoyer leurs articles autour des questions suivantes:

8 Comment participer à la délocalisation de l'art électronique et du bio-art produit en Amérique latine?

9 Comment proposer des lectures de "positionnalités" non-essentialistes d'Amérique latine, traversées à la fois par le "glocal » et le «translocal », comme par les nouvelles ontologies qui affectent tout ce qui existe au-delà de l'Amérique latine?

10 Comment se développent les limites ou les bords de l'art électronique ou l'émergence de l'art par rapport à la science et à la technologie, en particulier lorsque celles-ci débordent de possibilités, d'ouvertures et de nouvelles connaissances?

11 Comment se croisent, en Amérique latine, l'imagination conceptuelle et la multiplicité des points de vue avec les réalités qui n'existent pas nécessairement dans d'autres contextes?

De quelles manières l'art, la science et la technologie sont traversées par des questions propres à notre monde contemporain, notamment ses aspects liés à des états permanents de situations d'urgence?

13 Comment l'art, la science et la technologie peuvent agir de manière à contribuer à la création de nouvelles écologies sociales, humaines et post-humaines?

Version complète en espagnol

\section{RÉSUMÉS}

Le présent dossier rassemble des articles prenant comme objet les arts électroniques et biomédiales latino-américains en articulation avec un certain nombre de concepts, tels que les délocalités, les translocalités et l'activisme. Ces concepts proposés questionnent à la fois les différentes relations possibles entre les pratiques artistiques et les contextes de production spécifiques et celles entre les pratiques artistiques et les contextes de réception des arts. Ces derniers sont traversés aussi bien par des positionnements "glocals" et "translocals" divers que par des mutations ontologiques que notre époque est en train de produire en relation - non plus avec des localisations régionales - mais avec le vivant, dans un sens plus large. Ce numéro 11 de la revue Artelogie, voudrait également convoquer des propositions artistiques sur l'art et la technologie en lien avec l'activisme impliqué dans la mobilisation et la création de nouvelles écologies sociales issues des actions collectives, relationnelles et collaboratives.

La presente dossier reúne artículos que tomaron como objeto las artes electrónicas y biomediales latinoamericanas bajo el cobijo de una serie de conceptos en articulación: deslocalidades, 
translocalidades y activismo. Tales conceptos llaman a discutir de qué manera las prácticas artísticas evidencian tanto su vinculación, por un lado, con contextos específicos de producción y recepción de las artes atravesados por posicionalidades glocales y translocales diversas; y por otro lado, con las mutaciones ontológicas y epistemológicas que nuestra época está produciendo en relación - ya no con localizaciones regionales - sino a nivel de lo viviente tecnológico o biológico en un sentido más amplio. Se busca incluso reunir propuestas artísticas en arte y tecnología en sus relaciones con el activismo implicado en movilizar y crear nuevas ecologías sociales a partir de acciones colectivas, relacionales y colaborativas.

\section{INDEX}

Palabras claves : deslocalidades, translocalidades, activismo, artes electrónicas, bioarte latino americanas

Mots-clés : délocalisations, translocalisations, activisme, arts numériques et biomédiales latinoaméricaines

Keywords : delocalizations, translocalizations, activism, latin american electronic art, and bioart

\section{AUTEURS}

\section{PAT BADANI}

Pat Badani est artiste-chercheur, éducateur, écrivain et éditeur qui puise dans les domaines de l'art, de la science et de la technologie. Badani a reçu une maîtrise en beaux-arts du School of the Art Institute of Chicago (SAIC) aux Etats-Unis, et sa trajectoire académique inclut: professeur de médias intégrés à l'école d'art de l'Illinois State University (ILSTU); Directrice intérimaire du programme d'études interdisciplinaires en arts médiatiques au Columbia College de Chicago; et Rédacteur en chef de Media-N Journal, le New Media Caucus, (EE. UU.). Elle siège au conseil d'administration d'ISEA International (International Symposium on Electronic Art).

\section{ILIANA HERNANDEZ GARCIA}

Docteur en Esthétique de l'Université de la Sorbonne Paris-I. Postdoctorat Senior en Philosophie des Sciences de l'Ecole Normale Supérieure / Institut d'Histoire et Philosophie des Sciences et Techniques. Directrice d'études avancés, invitée par la Maison des Sciences de l'Homme à Paris. Professeure Titulaire du Département d'Esthétique de la Faculté d'Architecture et Design de la Pontificia Universidad Javeriana / Bogotá. Directrice de l'équipe de recherche en Esthétique, nouvelles technologies et habitabilité. Directrice de la collection d'édition entitulée Esthétique contemporaine et professeure du Doctorat en Sciences Sociales et Humaines. Chercheuse en Esthétique des arts éléctroniques et biomediales, vie artificielle et mondes virtuels immersives.

\section{CLAUDIA KOZAK}

Docteur en Lettres (UBA).Chercheur CONICET/Institut de Recherche "Gino Germani" (UBA). Professeur Titulaire, Faculté de Sciences Sociales et Professeur Adjoint, Faculté de Philosophie et Lettres, Université de Buenos Aires. Directrice de Ludión. Exploratorio latinoamericano de poéticas/políticas tecnológicas (http://www.ludion.org), coordinatrice Red de Literatura Electrónica Latinoamericana (http://litelat.net/). Elle intègre le Conseil de Directeurs de la Electronic Literature Organization (https://eliterature.org/) . Certains de ses livres sont : Poéticas/políticas tecnológicas en Argentina -1910-2010-; Tecnopoéticas argentinas. Archivo blando de arte 
y tecnología; Deslindes. Ensayos sobre la literatura y sus límites en el siglo XX, Contra la pared. Sobre graffitis, pintadas y otras intervenciones urbanas.

\section{PRISCILA ARANTES}

Priscila Arantes é pesquisadora e curadora em arte e estética contemporânea. É pós doutora pela Penn State University (USA) e doutora em Comunicação e Semiótica pela PUC/São Paulo. É professora titular de Arte e Tecnologia do Doutorado em Design da Universidade Anhembi Morumbi e Vice-coordenadora do Curso de Arte: história, crítica e curadoria da PUC/SP. É diretora artística e curadora do Paço das Artes, instituição da Secretaria de Estado da Cultura desde 2007.Entre seus livros destacam-se: Arte@Midia: perspectivas da estética digital (2012, 2ed.) e Reescrituras da Arte contemporânea: história, arquivo e mídia (2015). Entre os prêmios recebidos destacam-se: Society of Latin American Studies (2016), Getty Foundation (2016) e o 48 Prêmio Jabuti (finalista) pela publicação Arte@Mídia. 